\title{
Numerical Approaches to Industrial Flow Optimization
}

\author{
Harvey M. Thompson \\ Professor of Computational Fluid Dynamics \\ Head of the School of Mechanical Engineering \\ University of Leeds, Leeds, LS2 9JT, UK
}

\begin{abstract}
Numerical optimization methods are being used increasingly with flow simulations to improve the performance of fluid flow systems. Through a series of cases studies, this presentation will describe how combining experimental, flow modelling and optimization methods enable practical flow systems to be designed effectively within feasible time-scales. A number of the limitations of current numerical approaches will be highlighted and important new research directions will be discussed.
\end{abstract}

